Volume 8, No.6, November - December 2019

International Journal of Advanced Trends in Computer Science and Engineering

Available Online at http://www.warse.org/IJATCSE/static/pdf/file/ijatcse08862019.pdf

https://doi.org/10.30534/ijatcse/2019/08862019

\title{
Brain Tumor segmentation using modified Fuzzy metric based Approach with Adaptive Technique
}

\author{
D. SHERLIN ${ }^{1}$ ， Dr. D. MURUGAN ${ }^{2}$ \\ ${ }^{1}$ Research Scholar, Department of Computer Science \& Engineering, Manonmaniam Sundaranar University, \\ Abishekapatti, Tirunelveli 627 012, Tamil Nadu, India,sherlin.sundar@gmail.com \\ ${ }^{2}$ Professor and Head, Department of Computer Science \& Engineering, Manonmaniam Sundaranar University, \\ Abishekapatti, Tirunelveli 627 012, Tamil Nadu, India,dhanushkodim@yahoo.com
}

\begin{abstract}
Medical image diagnosis field has boomed up to be the key research area and this helps to achieve many advancement in diagnosing and treating patients. One such remarkable area is automatic brain tumour segmentation. Manual identification of tumor from MRI images are challenging, tedious and complex process and they require the support of skilled medical practitioner whereas this automatic segmentation helps in easy diagnosis of the tumor location exactly with less time and modelled to be user friendly. There are numerous automatic segmentation methods used for detecting the position of tumors. But accuracy and time are the major parameters which decide the priority and prominence of the segmentation algorithm. In our proposed modified adaptive $\mathrm{K}+\mathrm{FCM}$ method, the tumor detection is done based on three important stages, where the first stage is pre-processing which proceed with the denoising process, second stage is segmentation which works on clustering and tumor segmentation and final stage is post-processing which operates on the repair and denoising of segmented tumors. The proposed adaptive filtered based modified K+ Fuzzy corner metric algorithm shows better time precision and accuracy when compared to the existing algorithms like random forest and PCA segmentation methods. The algorithm is analysed with BRATS2013 datasets and results obtained shown that the proposed algorithm shows better segmentation.
\end{abstract}

Key words:Adaptive $\mathrm{K}+\mathrm{FCM}$, Brain Tumor segmentation, Principal Component Analysis(PCA), Random Forest Algorithm(RFA)

\section{INTRODUCTION}

Brain tumors are a group of abnormal tissue cells which grow uncontrollably and irregularly in shape, size and position. The existence of these type of tumors have major possibilities of affecting surrounding healthy tissues or cells which may in long run leads to the death of the patient. Brain tumours aremetastatic or primary and benign or malignant. The metastatic tumor in brain is a kind of cancer cell that banquet from other parts of body to the brain[1].Earlier detection of this kind of tissues and effective medical treatment may increase the rate of survival of the patient. The existence of brain tumor can be detected with help of MRI scan images. Magnetic Resonance Imaging (MRI) is based on computer system technology to generate the MRI scan report and display the digital image representation of the inner parts of the body. Brain tumor segmentation method is a one of the most significant and complicated in the field for medical applications because it requires identification of tumors from large amount of data. Segmentation have most significant process for the medical field image analysis [2]. In an object of detect the tumour density based segmentation of image [3], texture -based, intensity-based and edge-based segmentation techniques are using segmenting the MRI image. Segmenting the images are following stages are pre-processing, image segmentation andsegmentation. Image enhancement for noise reduction then extraction of layer interest are task of performed in pre-processing and segmentation. RI is generally more useful and accurate information over tumor type and position and size. Segmentation process can be performed automatically on various segmentation methods based on edges, pixels, region of tumors, threshold values and morphological techniques that helps to locate the position of tumors [4]. Classifying of the tumor portion using unsupervised and supervised learning methods can also be done with the help of clustering the image based on the White Matter (WM) and Gray Matter (GM) and others. The existing systems considered for the classification of brain tumor in our work are based on Support vector Machine (SVM) and Random Forest Algorithm (RFA) that are briefly explained in section 3. In our proposed technique, we have implemented modified fuzzy corner metric based clustering method which provides a best and most useful method for the classification of tumor locations. Fuzzy C- Mean clustering method and its various modified algorithms are used majorly in the field of classification, pattern identification, segmentation of images and collection and segregation of data. Clustering is a method used for pattern identification in an image when being processed for a huge amount of data computation. Input images of brain tumors are obtained from BRATS 2013 
dataset and conversion of dataset has been done with the help of 3D slicer tool which is briefly explained in section 3 .

\section{RELATED WORK}

UmitIlhan et al. (2017) [5] describes MRI image based techniques used to detect the portion of tumor in the brain. By applying the image processing technique on brain tumor detection used for better quality of the image. In this work discussed about morphological operations, threshold based pixel subtraction segmentation and image filtering techniques are used. This work obtaining clear images of the skull and brain tumor. M. Kazi et al. (2017) [6] presents of brain MRI segmentation is to specifically identify the tissue structure in the image volume. In this study implemented a new approach on adaptive thresholding and $\mathrm{K}+$ mean clustering algorithm, which is used to get Gray Matter (GM), White Matter (WM) and others. Meet Ozaet al. (2015) [7] discussed about the 3D slicer, using segmented images divided into gray and white matter using Random Forest Algorithm. Then by detecting the true and false patterns in brain tumor, the tree is designed based on the selected true nodes. AthulSukumar A M et al. (2017) [8] discussed about image processing, a feature extraction might be a special type of brain tumor reduction. The aim of the paper is to render a peculiarity in reduction and extracted features using PCA and classifying the given tomography image are benign or malignant using SVM. Abhishek Bal et al (2018)[9] Segmentation of Tumors from MRI image is challenging but time consuming as it is manually performed by field expertise medical practitioner .In his work he focused on new algorithm which is fuzzy c means algorithm to diagnose accurately the region of cancer. In the first step it proceeds by noise filtering later applying FCM algorithm to segment only tumor area .In this research multiple MRI images of brain can be applied detection of gliomas (Tumor) growth by advanced diameter technique.

\section{SEGMENTATION METHODOLOGIES}

Brain tumor segmentation is one of the most important and complex tasks in medical applications because it usually associated as huge amount of data. Brain tumor is dividing as full image region into sub regions such that are white matter (WM), gray matter (GM) and cerebrospinal fluid (CSF) spaces of the brain automated picture of various image components. Four popular MRI modalities used for gliomas diagnosis consist of T1-weighted MRI (T1), T2-weighted MRI (T2), T1-weighted MRI with gadolinium assessment enhancement (T1-Gd) and Fluid Attenuated Inversion recovery (FLAIR) During MRI acquisition process from training data set taken from Brats data set, various segmentation process following on.

\subsection{Principal Component Analysis (PCA) Algorithms}

The Brain tumor have segmented as MRI image as input and is taken from Brats data set. Principal Component Analysis(PCA)is used for the process of feature reduction and extraction from the training dataset which are converted to image format using 3D slicer. From this feature extracted image, SVM classification is performed and accuracy is evaluated. Initially give the input as MRI image and has been pre-processed as removal of noise using adaptive filter technique, segment the portion of tumor and the feature extraction is done by using PCA. It is used for reducing the dimensionality (size) of the data and the dimensionality reduction technique have transformed as vector $\omega 1$ to a vector $\$ 1$ has a dimensionality, Next, reduction of feature by using SVM classification [12] and it used for evaluate the accuracy. Detect the portion of brain tumor using SVM classifier and it measure the accuracy of the tumor and its computation time.

\subsection{RandomForest Algorithm (RFA)}

RFA mostly used for classify the volume of pixels in skull. RFAis same as tree, if it chosen only one feature for dimension then got the accuracy of detecting the tumor portion. The training data (Brats dataset) is processed into tree roots then it passes through every internal sub node.[6] Each test point have trained ata separately and pushed against all the node of trees and randomly choose the point during training process then make every nodes of tree unique by improving the generalization of imaginary data..The input MRI scan image taken from 3D slicer as Brats data set. The training data is a normal brain image and tumour is a group of abnormal cells in the brain.RDF can manage large databases and it control thousands of variables to estimates variable used for segmentation. Random forest classification to constructing the trained dataset for each trees. In this classification separate the tumor and brain portion. In this segmentation using detect the brain.

\subsection{Proposed methodology}

We proposed a modified adaptive $\mathrm{K}+$ fuzzy corner metric(adaptive $\mathrm{K}+\mathrm{FCM}$ ) segmentation methodology for the automatic segmentation of brain tumor. The proposed workflow and algorithm has been explained in figure 1 and 2.

\subsubsection{Adaptive Denoising}

Image acquisition of pre-processing technique for removing the noise using adaptive wiener threshold method. In this method using eliminate the Gaussian noise. Brain surface striping is the process of morphological technique with operates on structuring element for sizing and shaping the image. The morphological image processing (MIP) using leave the small area and leave the large are for erosion and dilation. In the brain surface striping based on morphological process for binary and grayscale image. In this brain surface striping depends on erosion based shrinking image size of small portion by using the below equation (1)

$$
\mathrm{B} \ominus \mathrm{S}=\{(\mathrm{x}, \mathrm{y}) \mid \mathrm{Sxy} \subseteq \mathrm{B}\}
$$

Here, B is a binary image and S represented as SE consist of ' 0 ' and ' 1 ' $2 \mathrm{~d}$ object component. $(\mathrm{x}, \mathrm{y})$ is the point of original 
location and After structure element is represented as Sxy finally, binary image $\mathrm{B} \ominus \mathrm{S}$ from eroding $\mathrm{B}$ by $\mathrm{S}$.

\subsubsection{Segmenting the Brain Tumor using Adaptive $\mathrm{K}+$ Fuzzy Corner Metric (adaptive $\mathbf{K}+\mathbf{F C M}$ )}

Segmenting the brain tumor based on adaptive $\mathrm{K}+\mathrm{FCM}$ is achieved using the clustering methodology. Adaptive $\mathrm{K}+$ algorithm that have initialize the centroid deterministically. The initial K-clustered centroids and the final K-cluster centroids are calculated using the modified $\mathrm{K}+$ algorithm where the fuzzy set [10] of $\mathrm{F}$ is mapped to the mapping set $\mathrm{K}$ to $[0,1]$ and mathematical representation of $\mathrm{F}: \mathrm{K} \rightarrow[0,1], \mathrm{x}$ $\rightarrow \mathrm{F}(\mathrm{x})$, here $\mathrm{F}(\mathrm{x})$ as a membership function of the fuzzy cluster set $\mathrm{K}$.

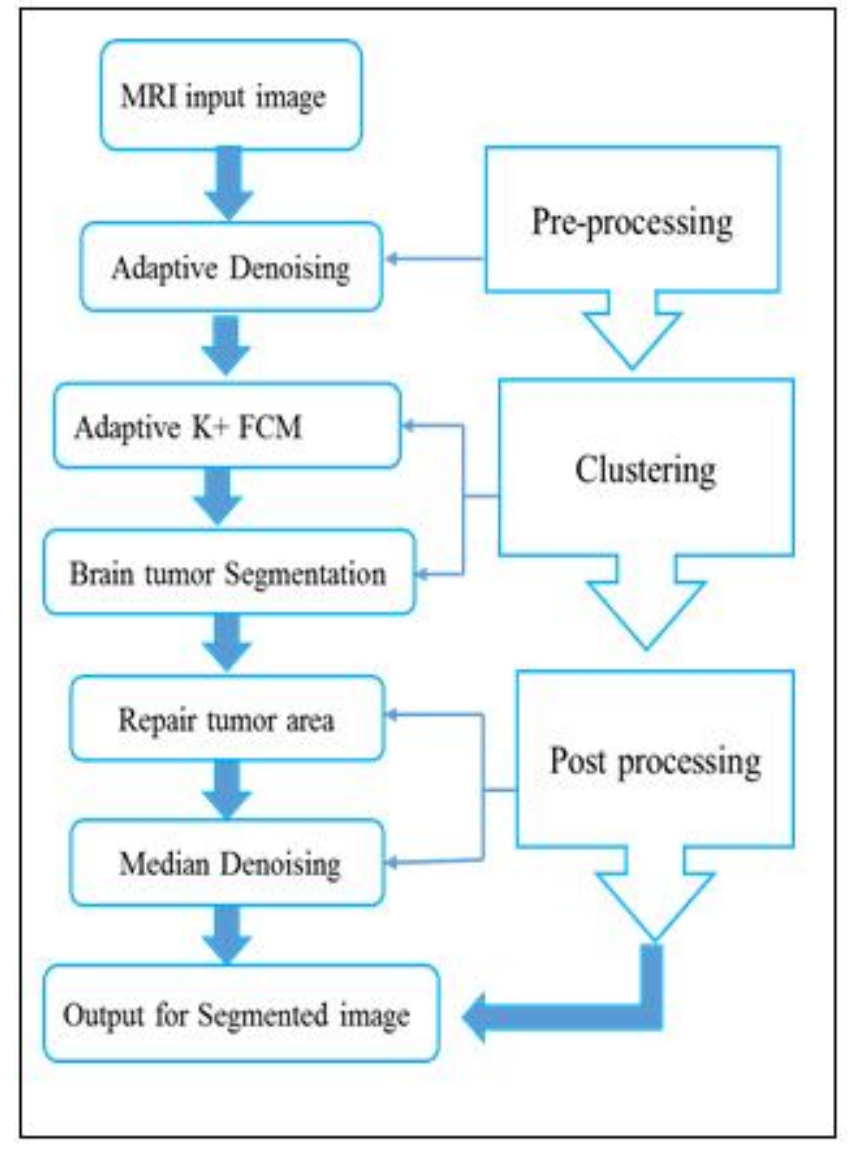

Figure 1.Proposed Methodology

\subsubsection{Adaptive K+FCM Algorithm}

The proposed $\mathrm{K}+\mathrm{FCM}$ algorithm depends on the fuzzy based clustering algorithm which introduces the Gaussian kernel for the efficient reduction of the sensitivity and improves the accuracy of the scaling parameter for this algorithm. Our proposed functionality clusters gray matter and white matter separately from the cerebrospinal fluid and finally segment the tumor areas. Here clustering of tumor areas are carried out with the help of thresholding.

\subsubsection{Repair Tumor area and Denoising the Image}

Detecting the portion of tumor based on repair tumor and denoising then will extract the tumor from brain. It is the process of histogram based thresholding technique and morphological operations. Median filter used for an alternative to the mean using a fixed threshold relative to the mean and median.

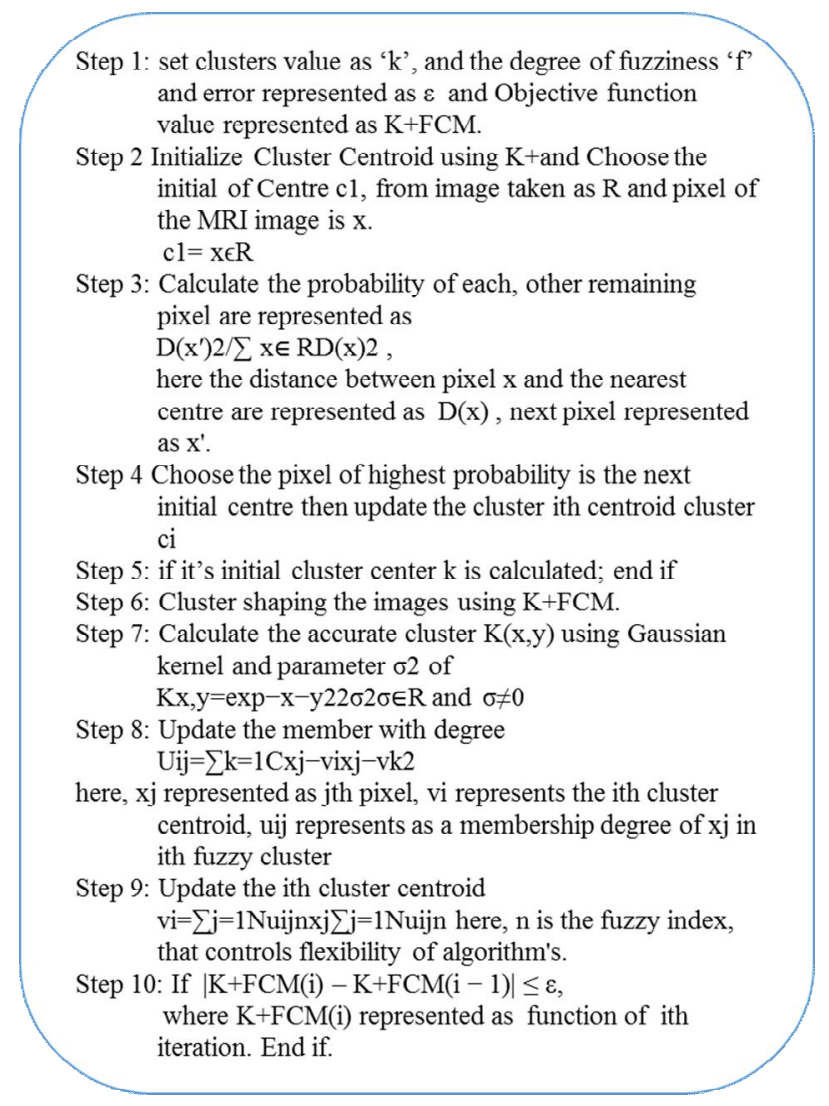

Figure 2.Proposed modified Algorithm

Median filter produce robust output is generated by using Gaussian filtering. The value of the result image is calculated based on equation (2) as T (n) if it's 1 for black 0 for white depending on whether it is ' $t$ ' percent darker than the average value of the previous s pixels.

$$
T(n)=\left\{\begin{array}{cc}
1 i f p_{n}<\left(\frac{t_{s}(\mathrm{n})}{s}\right)\left(\frac{100-t}{100}\right) \\
0 \quad \text { atherwise }
\end{array}\right.
$$

Here,'fs' is sum of the value of s pixels at the point $\mathrm{n}$ and $\mathrm{Pn}$ is called value of the pixel point $\mathrm{n}$. After the post processing, the repair works are carried out like filling small holes and filtering non-segmented areas from the extracted tumor areas

\section{BRAIN TUMOR RESULT ANALYSIS}

\subsection{MRI image as input}

In this paper taken an input from the Bratsmedical dataset on scan MRI Image in the year of 2013 brain tumor patient dataset and the image converting the scan image into gray scale image then further process of ground truth as detecting the brain tumor using PCA, RDF and proposed adaptive based $\mathrm{K}+\mathrm{FCM}$ segmentation algorithm. 


\subsection{Result for proposed adaptive based K+ FCM Segmentation algorithm}

Adaptive basedK+ FCM segmenting the MRI image and the image filtered as removing the noise using adaptive wiener Threshold technique. Median filter using control the noise that's helped as extract the tumor from brain. Brain surface extracting the image is the process of extracting the tumor from binary image.Erosion method used for shrinking the small portion of brain tumor then separated the white matter and gray matter in the binary image. Figure 3(a),(b)Adaptive $\mathrm{K}+\mathrm{FCM}$ clustering used for cluster the MRI image as 0 (white) and 1(black).In this clustered image post processing of repair the tumor area with help of morphological process and median filter using filter the tumor image.

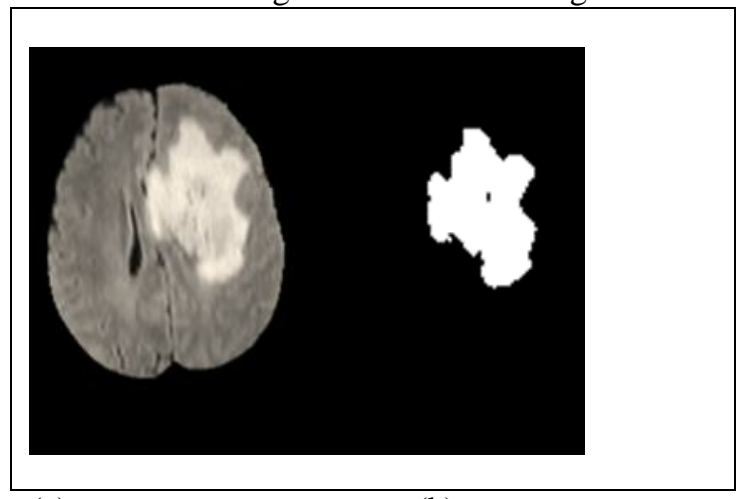

(a)

(b)

Figure 3: a)Input imageb) adaptive K+FCM Segmented image

\subsection{Comparison Result for RFA, PCA and adaptive K+FCM Algorithms}

Compare the following three algorithm such as RFA and PCA are existing system algorithm and adaptive $\mathrm{K}+\mathrm{FCM}$ is proposed system algorithm both algorithm have calculated as time duration for extracting the tumor portion of white matter and calculating the accuracy of existing system and proposed system. In this result analysis for detecting the exact tumor portion less time duration to predict the tumor in our proposed system adaptive K+FCM. Accurately to detect the white tumor is be better existing system. Comparison of RFA, PCA algorithm and adaptive $\mathrm{K}+\mathrm{FCM}$ algorithm is as less time duration with other algorithm.

Table 1:Comparison table of Time duration and accuracy for RFA, PCA and adaptive K+FCM Algorithm

\begin{tabular}{|l|l|l|l|l|l|l|}
\hline $\begin{array}{l}\text { S. } \\
\text { no }\end{array}$ & $\begin{array}{l}\text { Input } \\
\text { Image }\end{array}$ & \multicolumn{3}{|c|}{$\begin{array}{l}\text { Brain tumor } \\
\text { Segmentation of } \\
\text { Time Duration }\end{array}$} & \multicolumn{2}{|c|}{ Accuracy } \\
\cline { 3 - 7 } & RFA & PCA & $\begin{array}{l}\text { Adapti } \\
\text { ve } \\
\text { K+FC } \\
\text { M }\end{array}$ & Existing & $\begin{array}{c}\text { Propos } \\
\text { ed }\end{array}$ \\
\hline 1. & & 3.32 & 6.3 & 0.89 & 45 & 70 \\
\hline
\end{tabular}

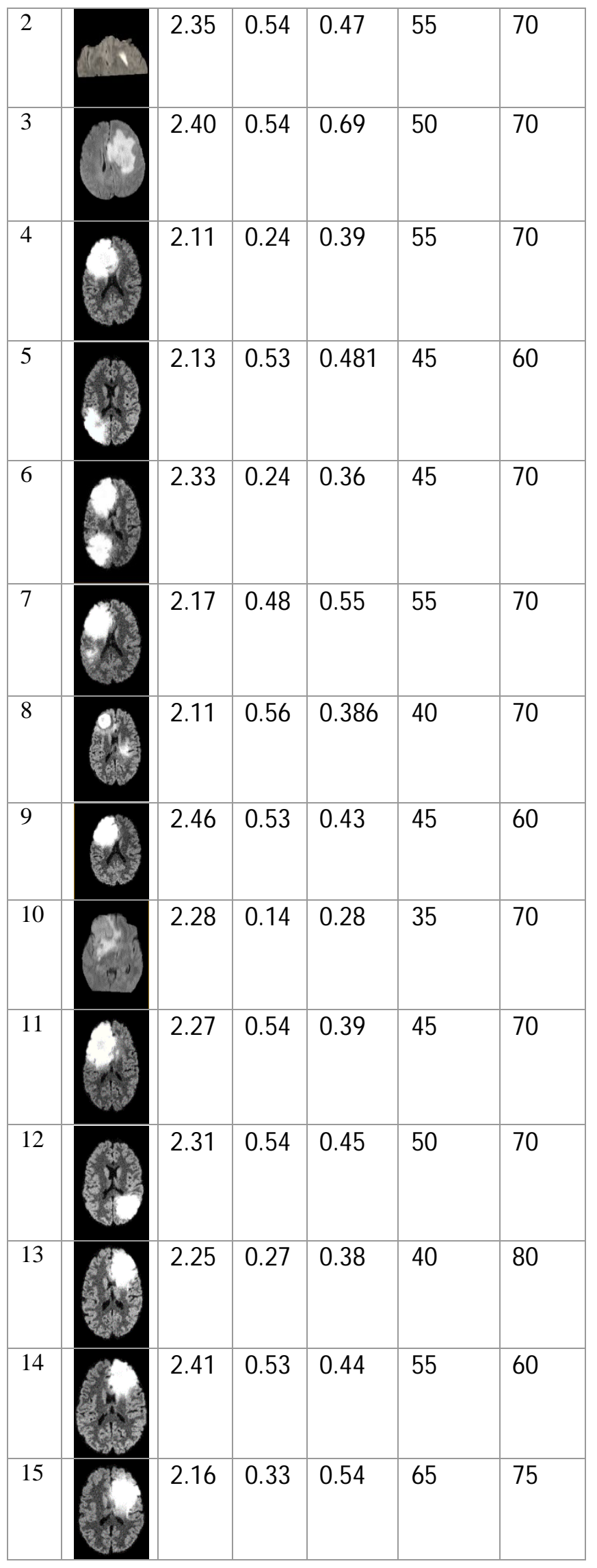




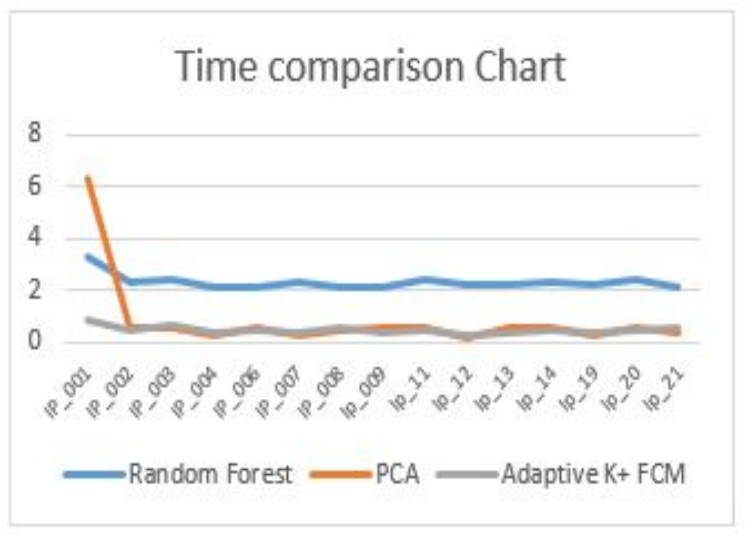

Figure 4:Comparison of RFA, PCA and adaptive K+RCM algorithm Time duration

Comparison chart explained about various MRI input image using detect the tumor portion for RFA, PCA and adaptive $\mathrm{K}+\mathrm{FCM}$ algorithm and our proposed system have provided high accuracy are shown in Table 1 and visual representation on figure 4 and 5 .

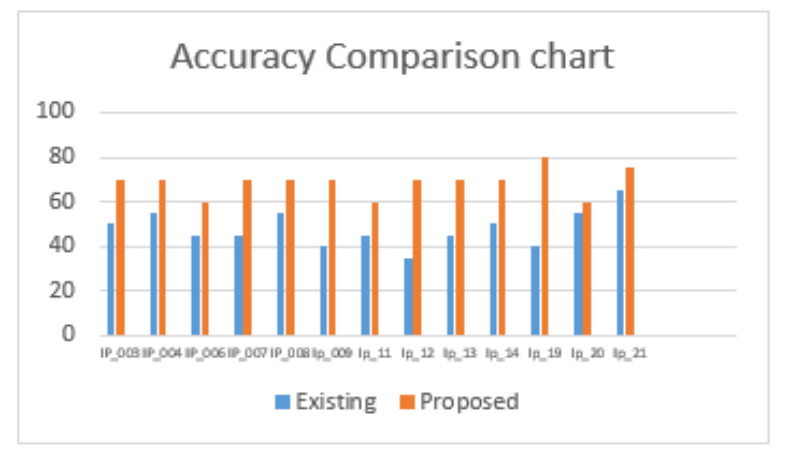

Figure 5:Comparison of existing and proposed system Accuracy

\section{CONCLUSION}

In our proposed system using adaptive $\mathrm{K}+\mathrm{FCM}$ algorithm which is based on modified fuzzy based segmentation algorithm, the detection of tumor location is accurate when compared to the existing algorithms. Though there are input images which shows the time consumption is high, segmentation by PCA algorithm observed is not correct and correlated with the Groundtruth image. Whereas in our proposed algorithm, the segmented tumor matches exactly with the ground truth image of BRATS dataset and the time consumption and accuracy is also improved when compared to the existing algorithms.

\section{REFERENCES}

1. Mohamed LamineToure "Advanced Algorithm for Brain Segmentation using Fuzzy to Localize Cancer and Epilepsy Region", International Conference on Electronics and Information Engineering (ICEIE 2010), Vol. no 2.

2. Wen, P, Zheng, L and Zhou, J, "Spatial credibilistic clustering algorithm in noise image segmentation", IEEE International Conference on Industrial Engineering and Engineering pp:309-315, 2005. Management, pp: 543 $-547,2007$. https://doi.org/10.1109/IEEM.2007.4419248

3. M. Schmidt, I. Levner, R. Greiner, A. Murtha, and A. Bistritz.'Segmentation using segmenting Brain Tumour in Alignment-Based Features".International Conference on Machine Learning and Applications, Los Angeles, December 2005.

4. UmitIlhana, Ahmet Ilhan" Brain tumor segmentation based on a new threshold approach" 9th International Conference on Theory and Application of Soft Computing, Computing with Words and Perception, ICSCCW 2017, 24-25 August 2017, Budapest, Hungary

5. I. M. Kazi , S. S. Chowhan, U. V. Kulkarn" MRI Brain Image segmentation using Adaptive Thresholding and K-means Algorithm” International Journal of Computer Applications (0975 - 8887) Volume 167 - No.8, June 2017 https://doi.org/10.5120/ijca2017914330

6. Meet Oza ,RupalKapdi "Brain Tumor Disease Identification Using Random Forest Classifiers" volume 7 March 2016 pp.202-204

7. AthulSukumar A M and Peter Augustine" Efficient Brain Tumor Classification using PCA and SVM" International Journal of Research in Engineering, IT and Social Sciences, ISSN 2250-0588, Impact Factor: 6.452, Volume 07 Issue 3, March 2017, Page 14-20

8. Gao J, Xie M: Skull stripping MR brain images using anisotropic diffusion filtering and morphological processing, Proc. International Symposium on Computer Network and Multimedia Technology, Wuhan 1:1-4,2009 https://doi.org/10.1109/CNMT.2009.5374500

9. Somasundaram K, Kalaiselvi T: Automatic brain extraction methods for $\mathrm{T} 1$ magnetic resonance images using region labeling and morphological operations. Comput Biol Med 41(8):2011

10.Juraj Horvath, 2006 "Image Fuzzy C-means" SAMI 2006.

11. Yoon UC, Kim JS, Kim IY, Kim SI. Adaptive fuzzy Cmeans for improved classification as a preprocessing procedure of brain parcellation. J Digit Imaging. 2001;14(2):238-240.doi: 10.1007/BF03190353.

12. Md. Al Mamun et al "Brain Tumor Detection Using Anisotropic Filtering, SVM Classifier and Morphological Operation From MR Images" International Conference on Computer, Communication, Chemical, Materials and Electronic Engineering. 0.1109/IC4ME2.2018.8465613. 